\title{
Overexpression of long non-coding RNA HOTAIR predicts a poor prognosis in patients with acute myeloid leukemia
}

\author{
SHENGHAO WU, CUIPING ZHENG, SONGYAN CHEN, XIAOPING CAI, \\ YUEJIAN SHI, BIJING LIN and YUEMIAO CHEN
}

\begin{abstract}
Department of Hematology, Wenzhou Central Hospital, Dingli Clinical College of Wenzhou Medical University, Wenzhou, Zhejiang 325000, P.R. China
\end{abstract}

Received October 1, 2014; Accepted June 16, 2015

DOI: $10.3892 / \mathrm{ol} .2015 .3552$

\begin{abstract}
The long non-coding RNA, HOX transcript antisense intergenic RNA (HOTAIR), has been indicated to have involvement in a number of cancers, however, its role in acute myeloid leukemia (AML) is unknown. The present study aimed to investigate the pattern of HOTAIR expression in AML and to evaluate its clinical significance in tumor progression. Quantitative polymerase chain reaction was performed to examine the HOTAIR expression in mononuclear cells from the bone marrow (BM) or peripheral blood specimens of 85 patients with newly diagnosed AML. The association of HOTAIR expression with the clinicopathological factors and prognosis of AML patients was statistically analyzed. The expression of HOTAIR was significantly upregulated in the AML patients compared with the healthy controls (mean expression value, $3.87 \pm 0.29$ vs. $1.28 \pm 0.09 ; \mathrm{P}<0.001$ ), and markedly decreased in the patients post-treatment compared with pre-treatment $(4.76 \pm 0.47$ vs. $2.81 \pm 0.27$; $\mathrm{P}<0.001)$. Moreover, high levels of HOTAIR were associated with higher white blood cell and BM blast counts $(\mathrm{P}<0.001$ and $\mathrm{P}=0.001$, respectively), and lower hemoglobin and platelet counts $(\mathrm{P}=0.007$ and 0.001 , respectively). Patients with a high level of HOTAIR expression had relatively poor overall survival (OS; 20.5 vs. 32.1 months, $\mathrm{P}=0.001$ ) and relapse-free survival (21.5 vs. 33.6 months, $\mathrm{P}=0.001$ ) times compared with those with a low level of HOTAIR expression. These data demonstrated that HOTAIR expression was upregulated in newly diagnosed AML patients and was associated with leukemic burden, and DFS and OS times. HOTAIR may represent a biomarker of a poor prognosis and is a potential therapeutic target for AML treatment.
\end{abstract}

Correspondence to: Dr Shenghao Wu, Department of Hematology, Wenzhou Central Hospital, Dingli Clinical College of Wenzhou Medical University, 2 Dajian Lane, Wenzhou, Zhejiang 325000, P.R. China

E-mail: shwu2014@163.com

Key words: long non-coding RNA, HOTAIR, acute myeloid leukemia, prognosis

\section{Introduction}

Acute myeloid leukemia (AML) is a heterogeneous hematopoietic malignancy characterized by the rapid accumulation and malignant proliferation of immature myeloid progenitors in the bone marrow (BM) and peripheral blood (PB) (1). Without treatment, AML quickly becomes fatal, and historically, it has always been associated with a poor prognosis. However, AML treatment has markedly improved over the last few decades, with improvements in risk assessment, post-remission chemotherapy and hematopoietic stem-cell transplantation $(2,3)$. However, even though complete remission (CR) is achieved after chemotherapy by the majority of AML patients, only $20 \%$ of obtain relatively long-term relapse-free survival (RFS) (4). Thus, in order to improve the diagnosis, prevention and treatment of this disease, detailed knowledge of the mechanisms that form the basis of AML development and progression must be acquired. Recently, it has been shown that long non-coding RNAs (lncRNAs) play a crucial role in hematopoietic differentiation and hematological malignancies, including AML (5).

Transcriptome analysis by tiling arrays and RNA sequencing has revealed that only $2 \%$ of the human genome is dedicated to the transcription of protein coding sequences and that $>90 \%$ of the genome is transcribed as non-coding RNAs $(6,7)$. IncRNAs are transcripts of $>200$ nucleotides and conventionally cannot be translated into proteins to participate in a large number of biological processes $(8,9)$. However, recent studies hypothesized that a number of IncRNAs are key developmental regulators that are involved in cell homeostasis and proliferation $(9,10)$. Notably, increasing numbers of studies are indicating that the abnormal expression of certain lncRNAs is associated with tumor growth, carcinogenesis or metastasis in a range of malignancies (11-15).

Hox transcript antisense intergenic RNA (HOTAIR) is a 2,158-bp lncRNA that is transcribed from the antisense strand of the homeobox $\mathrm{C}$ gene locus of chromosome 12. HOTAIR coordinates with chromatin-modifying enzymes and regulates gene silencing (16). Several recent studies have identified the aberrant expression of HOTAIR in a number of cancer types, including breast, colon, bladder, renal, pancreatic, cervical and lung cancer, and a high level of HOTAIR expression has been correlated with enhanced breast, colon and gastric cancer 
metastasis. In addition, HOTAIR-knockdown is able to inhibit the invasion and proliferation of cells, as well as altering cell cycle progression and inducing cells apoptosis, thus indicating that HOTAIR may function in the modulation of cancer progression (17-19).

To the best of our knowledge, no previous studies exist concerning the expression status, prognostic value and role of HOTAIR in AML. Thus, the aim of the present study was to investigate the correlation of HOTAIR expression with clinicopathological features and the prognosis of the patients with AML. The findings may improve our understanding of the roles and the clinic implications of HOTAIR in the development and progression of AML.

\section{Patients and methods}

Patients and specimens. This study was approved by the Research Ethics Committee of Wenzhou Central Hospital (Wenzhou, Zhejiang, China). Written informed consent was obtained from all patients according to the committee's regulations. Between February 2011 and August 2014, 85 patients in the Department of Hematology (Wenzhou Central Hospital) were diagnosed with AML according to the French-American-British (FAB) criteria (20). The cohort consisted of 45 males and 40 females, with a medium age of 45.2 years (range, 19.3-72.4 years). The median leukocyte count at diagnosis was 52,897/ $\mu 1$ (range, 793-327,100/ $\mu \mathrm{l}$; normal range, $4,000-11,000 / \mu 1$ ). Of the 85 enrolled patients, 5 patients presented with AML of type M1, 30 of type M2, 12 of type M4, 32 of type M5 and 6 of type M6, according to the World Health Organization classification system (21). The clinical characteristics of all the AML patients are summarized in Table I. A total of 66 patients received standard cytarabine (100 mg/m² daily, days 1-7) plus daunorubicin (45 mg/m² daily, days 1-3) 7+3 induction chemotherapy. Specimens were obtained from the BM or PB (peripheral white blood cells, $>50 \times 10^{9}$ ) of the patients at the time of diagnosis, and from 33 patients who were in CR following two cycles of chemotherapy. Additionally, 40 PB specimens were obtained from healthy donors as negative controls. Patients who achieved CR were then administered high- or medium-dose cytarabine-based chemotherapy for consolidation according to their physical condition. The 33 patients with CR were followed up for a median time of 22 months (range, 9-40 months), and the data were censored when the patients relapsed or succumbed.

RNA extraction and reverse transcription-quantitative polymerase chain reaction (RT-qPCR). Mononuclear cells from $\mathrm{PB}$ or BM specimens were isolated by Ficoll density gradient centrifugation (400 x g, 30 min; Hao Yang Biological Manufacture, Tianjin, China), and then washed and pelleted. Additionally, PB cluster of differentiation (CD) $34^{+}$cells from healthy donors were obtained using magnetic bead separation (EasySep Human CD34 Positive Selection kit; Stem Cell Technologies, Vancouver, BC, Canada). Total mRNA was extracted using TRIzol reagent (Invitrogen Life Technologies, Grand Island, NY, USA) according to the manufacturer's instructions. The quality and concentration of RNA were determined using a Nanodrop 2000 (Thermo Fisher Scientific Inc., Wilmington,
Table I. Clinicopathological variables of 85 patients with newly diagnosed AML divided into low $(n=52)$ and high $(n=33)$ HOTAIR expression groups.

\begin{tabular}{|c|c|c|c|c|}
\hline \multirow{2}{*}{$\begin{array}{l}\text { Clinicopathological } \\
\text { variables }\end{array}$} & \multirow{2}{*}{$\begin{array}{l}\text { No. of } \\
\text { patients }\end{array}$} & \multicolumn{2}{|c|}{ HOTAIR expression } & \multirow[b]{2}{*}{ P-value } \\
\hline & & High & Low & \\
\hline Age, years & & & & 0.950 \\
\hline$\leq 60$ & 39 & 15 & 24 & \\
\hline$>60$ & 46 & 18 & 28 & \\
\hline Gender & & & & 0.259 \\
\hline Male & 45 & 20 & 25 & \\
\hline Female & 40 & 13 & 27 & \\
\hline $\mathrm{WBC}, \mathrm{x} 10^{9} / 1$ & & & & 0.000 \\
\hline$<10$ & 50 & 9 & 41 & \\
\hline$\geq 10$ & 35 & 24 & 11 & \\
\hline HGB, g/dl & & & & 0.007 \\
\hline$<80$ & 49 & 25 & 24 & \\
\hline$\geq 80$ & 36 & 8 & 28 & \\
\hline PLT, $x 10^{9} / 1$ & & & & 0.001 \\
\hline$<50$ & 46 & 25 & 21 & \\
\hline$\geq 50$ & 39 & 8 & 32 & \\
\hline Blasts in BM, \% & & & & 0.001 \\
\hline$<50$ & 38 & 7 & 31 & \\
\hline$\geq 50$ & 47 & 26 & 21 & \\
\hline FAB subtype & & & & 0.347 \\
\hline $\mathrm{M} 1 / \mathrm{M} 2$ & 35 & 17 & 18 & \\
\hline M4/M5 & 44 & 13 & 21 & \\
\hline Other & 6 & 3 & 3 & \\
\hline Complete remission & & & & 0.082 \\
\hline Yes & 33 & 9 & 24 & \\
\hline No & 52 & 24 & 28 & \\
\hline
\end{tabular}

HOTAIR, HOX transcript antisense intergenic RNA; WBC, white blood cell; HGB, hemoglobin; PLT, platelet.

DE, USA). Next, $1 \mu \mathrm{g}$ total RNA was reverse transcribed from each sample to synthesize cDNA using the RT reagent kit (Fermentas, Glen Burnie, MD, USA) according to the manufacturer's instructions. qPCR was performed using the ABI 7300 Sequence Detection System with primer pairs and SYBR Green PCR Master Mix (Applied Biosystems, Foster City, CA, USA). The primer sequences used were as follows: HOTAIR forward, 5'-CAGTGGGGAACTCTGACTCG-3' and reverse, 5'-GTGCCTGGTGCTCTCTTACC-3'; $\beta$-actin forward, 5'-CACCATTGGCAATGAGCGGTTCC-3' and reverse, 5'-GTAGTTTCGTGGATGCCACAGG-3'. The amplification profile was $95^{\circ} \mathrm{C}$ for $5 \mathrm{~min}$, followed by 42 cycles of denaturation at $95^{\circ} \mathrm{C}$ for $15 \mathrm{sec}$, then annealing and extension at $60^{\circ} \mathrm{C}$ for $60 \mathrm{sec}$. The comparative $\mathrm{Ct}$ method $(\Delta \Delta \mathrm{Ct})$ was used for the quantification of gene expression. The relative expression of HOTAIR to $\beta$-actin was calculated using the equation $2^{-\Delta \Delta \mathrm{Ct}}$, where $\Delta \mathrm{Ct}=\mathrm{Ct}_{\text {HOTAIR }}-\mathrm{C}_{\mathrm{t} \beta \text {-actin }}$. Each sample was analyzed in triplicate and the mean expression level was calculated. 
Statistical analysis. Statistical analysis was performed with SPSS 16.0 for Windows (SPSS Inc., Chicago, IL, USA). Data are expressed as the mean \pm standard deviation. The Kruskal-Wallis non-parametric test was used to evaluate the difference in HOTAIR expression between the AML patients and the healthy controls. The paired t-test was used to evaluate the difference in HOTAIR expression prior to and following chemotherapy. Pearson's $\chi^{2}$ test was used to evaluate the association between HOTAIR expression and clinicopathological characteristics. Survival curves were plotted using the Kaplan-Meier product-limit method, and differences between survival curves were tested using the log-rank test. RFS was defined as the time between the achievement of CR and the time of the hematological relapse or the last follow-up. Overall survival (OS) was defined as the time between the moment of diagnosis and mortality or the last follow-up. Cox regression analysis in a forward stepwise method was used to evaluate the effect of multiple independent prognostic factors on survival outcome. Differences were considered to be statistically significant when $\mathrm{P}<0.05$.

\section{Results}

HOTAIR is overexpressed in AML patients. The HOTAIR expression levels were detected in $\mathrm{BM} / \mathrm{PB}$ samples from the patients with AML and the healthy controls by RT-qPCR. As shown in Fig. 1A, the expression of HOTAIR was significantly upregulated in the AML patients compared with the healthy controls (mean expression value, $3.87 \pm 0.29$ vs. $1.28 \pm 0.09$; $\mathrm{P}<0.001)$. Additionally, the $33 \mathrm{AML}$ patients who achieved CR following one or two cycles of chemotherapy were monitored for HOTAIR expression during the course of treatment. The mean expression value of these AML patients markedly decreased when $\mathrm{CR}$ was achieved after chemotherapy (mean expression value, $4.76 \pm 0.47$ vs. $2.81 \pm 0.27 ; \mathrm{P}<0.001$ ).

Correlations between the expression of HOTAIR and the clinicopathological factors in AML patients. To identify the clinical relevance of HOTAIR expression in AML patients, the correlation between HOTAIR expression and clinicopathological parameters was assessed. Those AML patients with HOTAIR expression levels at less than the median value (3.87) were assigned to the low expression group (mean expression value, $1.98 ; \mathrm{n}=52$ ), and those with expression above the median value were assigned to the high expression group (mean expression value, $6.84 ; n=33$ ). As shown in Table I, high levels of HOTAIR were associated with higher white blood cell and BM blast counts $(\mathrm{P}<0.001$ and $\mathrm{P}=0.001$; respectively), and a lower hemoglobin level and platelet count $(\mathrm{P}=0.007$ and 0.001 ; respectively). However, other clinical characteristics, including age, gender and FAB subtype were not directly associated with the high expression of HOTAIR.

Association between HOTAIR expression and clinical outcomes of AML patients. In total, 66 patients received standard induction chemotherapy, The CR rate following two cycles of chemotherapy was $27.3 \%$ (9/33) in the high expression group compared with $46.2 \%$ (24/52) in the low expression group $(\mathrm{P}=0.082)$. Despite the high $\mathrm{CR}$ rate in the low expression group, there was no statistically significant
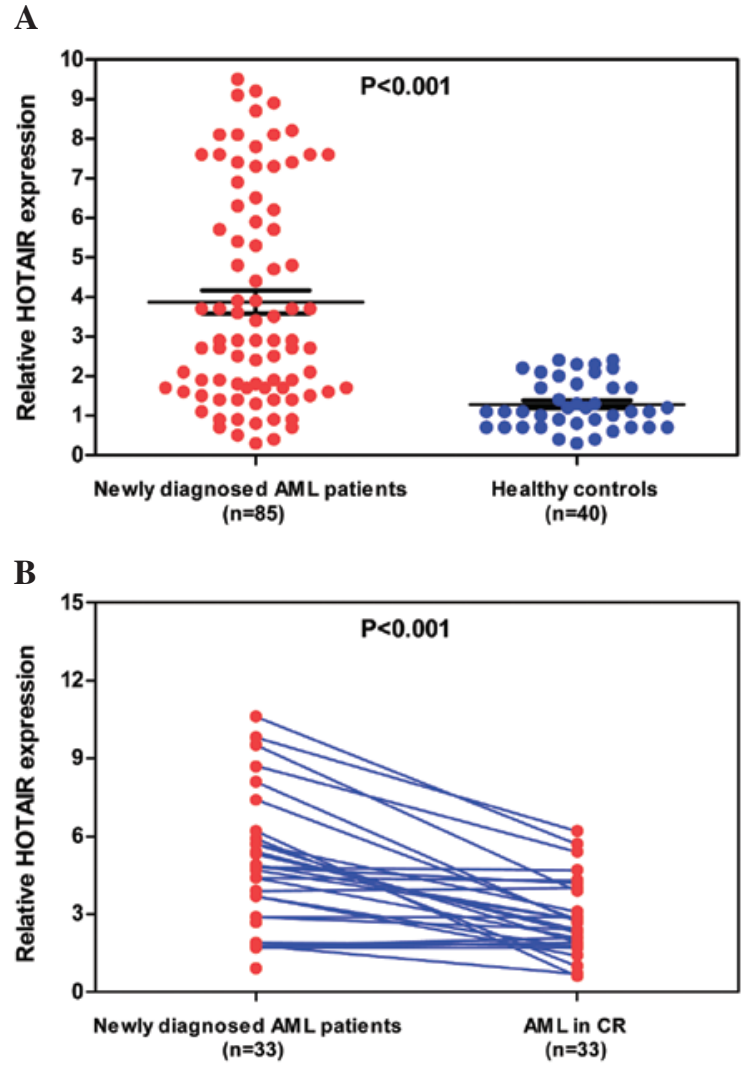

Figure 1. HOX transcript antisense intergenic RNA (HOTAIR) expression in newly diagnosed acute myeloid leukemia (AML) patients and healthy controls, as detected by reverse transcription-quantitative polymerase chain reaction analysis. (A) Expression levels of HOTAIR in newly diagnosed AML patients and healthy controls. (B) Expression levels of HOTAIR in 33 AML patients prior to and following complete remission (CR).

difference between the values of the two groups $(\mathrm{P}=0.082)$. The 33 patients who achieved a CR were followed up for a median time of 22 months (range, 9-40 months). OS and RFS survival curves in high expression and low expression groups are shown in Fig 2. Patients with high HOTAIR expression exhibited significantly poorer OS (20.5 vs. 32.1 months; $\mathrm{P}=0.001)$ and RFS (21.5 vs. 33.6 months; $\mathrm{P}=0.001)$ times compared with those with low HOTAIR expression. In multivariate analysis, the Cox regression analysis revealed that HOTAIR overexpression was an independent prognostic factor for OS (hazard ratio, 3.37; 95\% confidence interval, 0.99-8.31; $\mathrm{P}=0.008$ ). Statistical values for HOTAIR expression and other clinical parameters derived from the Cox stepwise proportional hazards model are presented in Table II.

\section{Discussion}

It is becoming evident that mammalian genomes encode thousands of lncRNAs, and multiple lines of evidence increasingly support the idea that certain lncRNAs could be used as biomarkers that predict the prognosis of tumor targets of human cancer $(22,23)$. HOTAIR was first identified as one of 231 lncRNAs that are associated with the human HOX loci, which binds to polycomb repressive complex 2 (PRC2), a transcriptional co-repressor, and recruits it to silence the target genes (18). HOTAIR is also able to interact with a second histone 
Table II. Cox multivariate analysis of factors for overall survival in AML patients.

\begin{tabular}{lccc}
\hline \multirow{2}{*}{ Factors } & \multicolumn{3}{c}{$95 \%$ confidence } \\
\hline WBC & Hazard ratio & interval & P-value \\
HGB & 0.87 & $0.44-1.39$ & 0.047 \\
PLT & 0.93 & $0.74-1.79$ & 0.088 \\
Blasts in BM & 2.07 & $1.13-3.62$ & 0.034 \\
Complete remission & 1.15 & $0.41-1.77$ & 0.078 \\
HOTAIR expression & 3.37 & $1.14-2.55$ & 0.031 \\
& & $0.99-8.31$ & 0.008 \\
\hline
\end{tabular}

WBC, white blood cell; HGB, hemoglobin; PLT, platelet; BM, bone marrow; HOTAIR, HOX transcript antisense intergenic RNA.

A



B

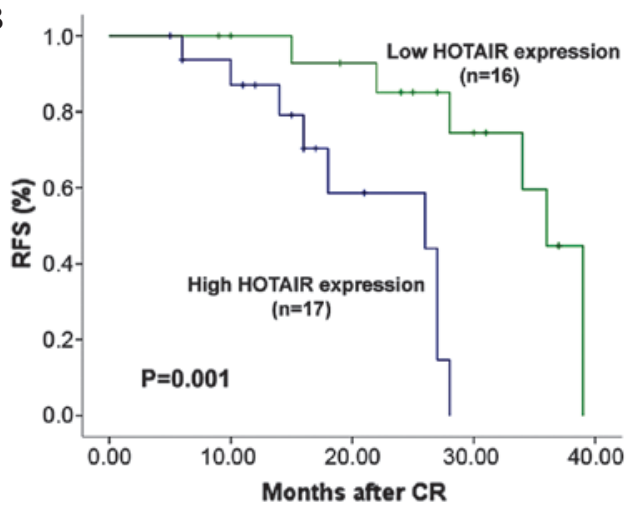

Figure 2. Kaplan-Meier survival curves for overall survival (OS) and relapse-free survival (RFS) according to HOX transcript antisense intergenic RNA (HOTAIR) expression from 32 AML patients in CR. (A) The OS rate of AML patients in complete remission (CR) with high or low HOTAIR expression. (B) The RFS rate of AML patients in CR with high or low HOTAIR expression. The P-value was calculated using the log-rank test.

modification complex, the LSD1/CoREST/REST complex, which functions by coordinating the targeting of PRC2 and LSD1 to chromatin for methylation of coupled histone H3K27 and demethylation of K4 (24). HOTAIR is believed to be an oncogene due to its elevated expression levels in a number of cancer types, and due to its ability to mediate the invasion and metastasis of cancer cells. For example, Huang et al revealed that HOTAIR expression in cervical cancer tissues was significantly upregulated compared with the matched non-tumorous tissues, and increased HOTAIR expression was significantly correlated with the International Federation of Gynecology and Obstetrics stage, lymph node metastasis, depth of cervical invasion and tumor size (25). HOTAIR was associated with the carcinogenesis and invasion of gastric adenocarcinoma, HOTAIR-targeted RNA interference can reduce the proliferation, invasion and migration abilities of gastric cancer cell lines (26). Similarly, dysregulation of HOXA5 expression has also been reported in association with tumorigenesis and progression in lung cancer (27-29). These observations suggest that HOTAIR has a direct role in the modulation of cancer progression and may be useful in patients with cancer as a novel prognostic or progression marker. However, in AML, the HOTAIR expression status and its prognostic roles are unclear.

In the present study, through the use of qPCR, it was confirmed for the first time that the expression of lncRNA HOTAIR was markedly unregulated in patients with newly diagnosed AML compared with healthy controls; these results were consistent with other studies regarding solid tumors. Moreover, the level of HOTAIR expression was significantly decreased following chemotherapy when patients achieved $\mathrm{CR}$, indicating that HOTAIR expression is consistent with tumor burden, and that HOTAIR expression can be used as a prognostic marker of relapse. In addition, the present results indicated that the upregulation of HOTAIR in AML patients was significantly correlated with higher white blood cell and BM blast counts, and a lower hemoglobin level and platelet count, which represented more aggressive clinicopathological features. Finally, AML patients with high HOTAIR expression tended to have poorer OS and RFS times compared with those with low HOTAIR expression, indicating that the expression of HOTAIR is significant in the classification of AML prognosis. Taken together, these data suggest that HOTAIR may function as an oncogene in the development of AML, and may represent a candidate prognostic biomarker for AML patients.

The aforementioned findings that HOTAIR overexpression was associated with aggressive tumor progression indicated that its possible prognostic value in AML patients should be investigated in the present study. According to the univariate and multivariate analyses, HOTAIR overexpression was identified as an independent predictor for the OS of AML patients, which was in agreement with recent findings in NSCLC and cervical cancer $(25,26)$, suggesting that the detection of increased HOTAIR expression may aid in the identification of AML patients with a poor prognosis, and could therefore be a novel prognostic marker for AML patients.

In summary, the present study provides evidence for the first time that HOTAIR may act as an oncogenic gene in AML, and that it may represent a potential biomarker of poor prognosis and a potential therapeutic target for AML intervention. However, the precise molecular mechanisms behind the involvement of HOTAIR in AML require further investigation.

\section{References}

1. Troy JD, Atallah E, Geyer JT and Saber W: Myelodysplastic syndromes in the united states: An update for clinicians. Ann Med 46: 283-289, 2014.

2. Daver N and Cortes J: Molecular targeted therapy in acute myeloid leukemia. Hematology 1 (17 Suppl) : S59-S62, 2012. 
3. Stone R, Sekeres M and Garcia-Manero G: Evolving strategies in the treatment of MDS and AML. Clin Adv Hematol Oncol 7: 1-14; quiz $12 \mathrm{p}$ following 14, 2009.

4. Tallman MS, Gilliland DG and Rowe JM: Drug therapy for acute myeloid leukemia. Blood 106: 1154-1163, 2005.

5. Fatica A: Noncoding RNAs in acute myeloid leukemia: From key regulators to clinical players. Scientifica (Cairo) 2012: 925758, 2012

6. ENCODE Project Consortium, Birney E, Stamatoyannopoulos JA Dutta A, Guigó R, Gingeras TR, Margulies EH, Weng Z, Snyder M, Dermitzakis ET, et al: Identification and analysis of functiona elements in $1 \%$ of the human genome by the ENCODE pilot project. Nature 447: 799-816, 2007.

7. Mattick JS: Non-coding RNAs: The architects of eukaryotic complexity. EMBO Rep 2: 986-991, 2001.

8. Wilusz JE, Sunwoo H and Spector DL: Long noncoding RNAs: Functional surprises from the RNA world. Genes Dev 23: 1494-1504, 2009.

9. Johnsson $\mathrm{P}$ and Morris $\mathrm{KV}$ : Expanding the functional role of long noncoding RNAs. Cell Res 24: 1284-1285, 2014.

10. Bhan A and Mandal SS: Long noncoding RNAs: Emerging stars in gene regulation, epigenetics and human disease. Chem Med Chem 9: 1932-1956, 2014.

11. Cheng W, Zhang Z and Wang J: Long noncoding RNAs: New players in prostate cancer. Cancer Lett 339: 8-14, 2013.

12. Xia T, Liao Q, Jiang X, Shao Y, Xiao B, Xi Y and Guo J: Long noncoding RNA associated-competing endogenous RNAs in gastric cancer. Sci Rep 4: 6088, 2014.

13. Zhou S, Wang J and Zhang Z: An emerging understanding of long noncoding RNAs in kidney cancer. J Cancer Res Clin Oncol 140: 1989-1995, 2014

14. Zhao J and Lawless MW: Long noncoding RNAs and their role in the liver cancer axis. Nat Rev Gastroenterol Hepatol 2013.

15. Tsai MC, Spitale RC and Chang HY: Long intergenic noncoding RNAs: New links in cancer progression. Cancer Res 71: 3-7, 2011.

16. Woo CJ and Kingston RE: HOTAIR lifts noncoding RNAs to new levels. Cell 129: 1257-1259, 2007.

17. Wu Y, Zhang L, Wang Y, Li H, Ren X, Wei F, Yu W, Wang X Zhang L, Yu J, et al: Long noncoding RNA HOTAIR involvement in cancer. Tumour Biol 35: 9531-9538, 2014.

18. Cai B, Song XQ, Cai JP and Zhang S: HOTAIR: A cancer-related long non-coding RNA. Neoplasma 61: 379-391, 2014.

19. Zhang J, Zhang P, Wang L, Piao HL and Ma L: Long non-coding RNA HOTAIR in carcinogenesis and metastasis. Acta Biochim Biophys Sin (Shanghai) 46: 1-5, 2014.
20. Bennett JM, Catovsky D, Daniel MT, Flandrin G, Galton DA, Gralnick HR and Sultan C: Proposed revised criteria for the classification of acute myeloid leukemia. A report of the French-American-British Cooperative Group. Ann Intern Med 103: 620-625, 1985.

21. Arber DA, Brunning RD, Le Beau MM, Falini B, Vardiman JW, Porwit A, Thiele J and Bloomfield CD: Acute myeloid leukaemia with recurrent genetic abnormalities. In: WHO classification of tumours of haematopoietic and lymphoid tissues. Swerdlow S, Campo E and Harris NL (eds). 4th edition. IARC Press, Lyon, France, pp110-123, 2008.

22. Guttman M, Amit I, Garber M, French C, Lin MF, Feldser D, Huarte M, Zuk O, Carey BW, Cassady JP, et al: Chromatin signature reveals over a thousand highly conserved large non-coding RNAs in mammals. Nature 458: 223-227, 2009.

23. Maruyama R and Suzuki H: Long noncoding RNA involvement in cancer. BMB Rep 45: 604-611, 2012.

24. Tsai MC, Manor O, Wan Y, Mosammaparast N, Wang JK, Lan F, Shi Y, Segal E and Chang HY: Long noncoding RNA as modular scaffold of histone modification complexes. Science 329: 689-693, 2010.

25. Huang L, Liao LM, Liu AW, Wu JB, Cheng XL, Lin JX and Zheng M: Overexpression of long noncoding RNA HOTAIR predicts a poor prognosis in patients with cervical cancer. Arch Gynecol Obstet 290: 717-723, 2014.

26. Lee NK, Lee JH, Park CH, Yu D, Lee YC, Cheong JH, Noh SH and Lee SK: Long non-coding RNA HOTAIR promotes carcinogenesis and invasion of gastric adenocarcinoma. Biochem Biophys Res Commun 451: 171-178, 2014.

27. Liu XH, Liu ZL, Sun M, Liu J, Wang ZX and De W: The long non-coding RNA HOTAIR indicates a poor prognosis and promotes metastasis in non-small cell lung cancer. BMC Cancer 13: 464, 2013

28. Ono H, Motoi N, Nagano H, Miyauchi E, Ushijima M, Matsuura M, Okumura S, Nishio M, Hirose T, Inase N, et al: Long noncoding RNA HOTAIR is relevant to cellular proliferation, invasiveness and clinical relapse in small-cell lung cancer. Cancer Med 3: 632-642, 2014.

29. Zhao W, An Y, Liang Y and Xie XW: Role of HOTAIR long noncoding RNA in metastatic progression of lung cancer. Eur Rev Med Pharmacol Sci 18: 1930-1936, 2014. 